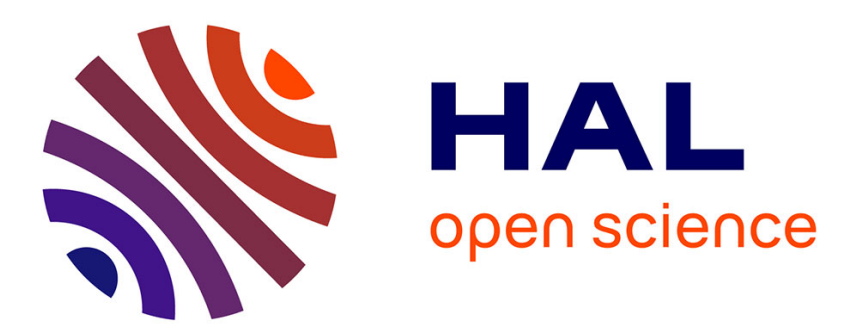

\title{
Second and third derivative of a tunnel junction characteristic application to the observation of electronic excitations
}

\author{
J. Klein, A. Léger, B. Delmas, S. de Cheveigne
}

\section{To cite this version:}

J. Klein, A. Léger, B. Delmas, S. de Cheveigne. Second and third derivative of a tunnel junction characteristic application to the observation of electronic excitations. Revue de Physique Appliquée, 1976, 11 (2), pp.321-325. 10.1051/rphysap:01976001102032100 . jpa-00244065

HAL Id: jpa-00244065

https://hal.science/jpa-00244065

Submitted on 1 Jan 1976

HAL is a multi-disciplinary open access archive for the deposit and dissemination of scientific research documents, whether they are published or not. The documents may come from teaching and research institutions in France or abroad, or from public or private research centers.
L'archive ouverte pluridisciplinaire HAL, est destinée au dépôt et à la diffusion de documents scientifiques de niveau recherche, publiés ou non, émanant des établissements d'enseignement et de recherche français ou étrangers, des laboratoires publics ou privés. 


\title{
SECOND AND THIRD DERIVATIVE OF A TUNNEL JUNCTION CHARACTERISTIC APPLICATION TO THE OBSERVATION OF ELECTRONIC EXCITATIONS (*)
}

\author{
J. KLEIN, A. LÉGER, B. DELMAS and S. DE CHEVEIGNE \\ Groupe de Physique des Solides de l'Ecole Normale Supérieure (**), \\ Tour 23, 2, place Jussieu, 75221 Paris, Cedex 05, France
}

(Reçu le 4 août 1975, accepté le 9 octobre 1975)

\begin{abstract}
Résumé. - La spectroscopie par effet tunnel dans le domaine des fortes polarisations est un outil intéressant pour l'étude des excitations électroniques dans les jonctions. Mais les courbes d'effet tunnel présentent alors des fonds continus très importants et cela constitue une des difficultés essentielles de ces études. Toutefois ce fond continu dépend beaucoup de la dérivée seconde qui est utilisée. Nous comparons les aptitudes des fonctions $\mathrm{d}^{2} V / \mathrm{d} I^{2}, \mathrm{~d}^{2} I / \mathrm{d} V^{2}$ et $1 / \sigma . \mathrm{d} \sigma / \mathrm{d} V$ quant à la détection d'un phénomène d'effet tunnel inélastique et concluons que la dernière est la mieux adaptée. Nous décrivons un montage qui permet d'obtenir cette fonction directement à la sortie d'une détection synchrone. Nous discutons ensuite la possibilité d'utiliser des dérivées d'ordre supérieur pour faire ressortir des structures très petites mais à variation relativement rapide et proposons un montage pour tracer une caractéristique de dérivée troisième de jonction tunnel. Pour illustrer les possibilités de cette méthode, nous nous intéressons à un cas où un pic d'effet tunnel inélastique attendu n'avait pas pu être observé jusqu'à présent du fait d'un fond continu trop important. La technique de la dérivée troisième nous permet de l'observer distinctivement.
\end{abstract}

Abstract. - Tunneling spectroscopy at high bias has proved to be useful áš a tool for studying electronic excitations in junctions although one major difficulty encountered is the huge background in the second derivative of the tunneling characteristics $I(V)$. This background 'however depends on which second derivative is used. We compare the sensitivity of the functions $\mathrm{d}^{2} V / \mathrm{d} I^{2}, \mathrm{~d}^{2} I / \mathrm{d} V^{2}$ and $1 / \sigma \cdot \mathrm{d} \sigma / \mathrm{d} V$ to inelastic phenomena and conclude that the last one is rather better adapted. We describe a system for obtaining this function at the output of a lock-in detector. The use of higher derivatives to show up very weak, but rather abrupt, peaks in the curves is discusséd and we propose a circuit to plot a third derivative characteristic of tunnel junctions. In order to illustrate the possibilities of this equipment, we re-examine a situation where a peak due to an inelastic phenomenon was expected but not yet observed because of a large base-line and where the use of the third dẻrivative technique enabled us to see it very distinctly.

1. Introduction. - Tunneling investigations [1] were originally made at low bias $(0.5 \mathrm{~V})$ where the conductance of the junctions could be considered to be constant in a first approximation. Any second derivative of the $I-V$ characteristic was then nicely adapted to the search for small non-linearities. In the high bias region now studied, the dynamical conductance $(\sigma)$ varies very rapidly with the $d$. c voltage, and the different second derivatives $\left(\mathrm{d}^{2} V / \mathrm{d} I^{2}, \mathrm{~d}^{2} I / \mathrm{d} V^{2}, 1 / \sigma . \mathrm{d} \sigma / \mathrm{d} V\right)$ are not at all equivalent : some have a large background that can hide the structure of interest. This if of special importance for the study of electronic transitions which generally occur for a bias higher than $1 \mathrm{~V}$ and explains

(*) Work supported, in part, by the D. R. M. E. contract $n^{\circ} 73 / 683$.

$(* *)$ Laboratoire associé au C. N. R. S. why an effect predicted in 1967 [2] was discovered only in 1972 [3]. In part 2 we discuss the ability o these different functions to bring out inelastic peaks and describe a system that gives the $1 / \sigma . \mathrm{d} \sigma / \mathrm{d} V$ characteristic.

Moreover, in order to observe a weak structure on curves, it may be useful to plot a third order derivative [3], as the higher the order of the derivative the better small bumps can be distinguished from the base line. Part 3 of the paper is devoted to third derivative systems.

Finally, in part 4 , an experiment is described to illustrate the possibilities of such systems.

2. Second derivative $\frac{1}{\sigma} \cdot \frac{d \sigma}{d V} \cdot-2.1$ ABILITY OF DIFFERENT SECOND DERIVATIVES TO BRING OUT INELASTIC 
PEAKS. - From an experimental point of view, the easiest second derivative to obtain is $\mathrm{d}^{2} V / \mathrm{d} I^{2}$, as a constant current modulation can be easily applied to the junction [4]. However, other functions may be better adapted to the tunneling spectroscopy.

In figure 1 , the possibilities of the $\mathrm{d}^{2} V / \mathrm{d} I^{2}$ characteristic are compared to those of the logarithmic deriva-

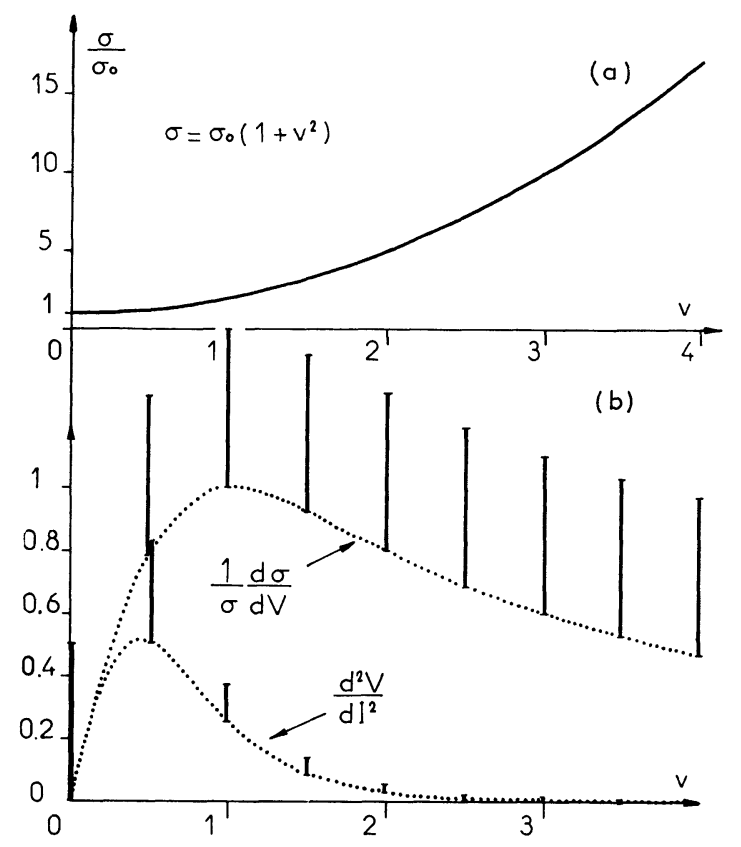

FIG. 1. - a) Model for a junction conductance that rapidly increases with voltage : $\sigma=\sigma_{0}\left(1+v^{2}\right)$ with $\left.v=V / V_{0} . b\right)$ Two second order derivatives of this junction characteristic $: \mathrm{d}^{2} V / \mathrm{d} I^{2}$ and $1 / \sigma . \mathrm{d} \sigma / \mathrm{d} V$. Besides the general variation of the curves, an inelastic process is supposed to occur that locally increases the conductance $\sigma(v)$ by a small fraction $\beta$ at a given voltage ( $\left.v_{\text {inel }}\right)$ according to eq. (1). The vertical lines indicate the amplitude of the corresponding peaks for different values of $v_{\text {inel }}(0,0.5,1$, $1.5, \ldots)$. When $v_{\text {inel }}$ increases this amplitude decreases very quickly for the $\mathrm{d}^{2} V / \mathrm{d} I^{2}$ derivative, whereas it remains constant for $1 / \sigma \cdot \mathrm{d} \sigma / \mathrm{d} V$. Clearly, this last function appears to be more adequate for detecting such inelastic peaks at high bias.

tive $1 / \sigma . \mathrm{d} \sigma / \mathrm{d} V$. For that purpose, we have used a model to simulate the conductance variation of the junction : it is assumed that $\sigma$ increases as the function $\sigma_{0}\left(1+v^{2}\right)$, where $v$ is a reduced voltage, $v=V / V_{0}$, $V_{0}$ being a distinctive parameter of the junction. Some inelastic process is supposed to occur at a given reduced bias $\left(v_{\text {inel }}\right)$ increasing the local conductance by a small factor $\beta$ :

$$
\sigma(v)=\sigma_{0}\left(1+v^{2}\right)\left[1+\beta Y\left(v-v_{\text {inel }}\right)\right],
$$

where $Y(v)$ is the unit step function. We study the two derivatives and the corresponding inelastic peaks for different values of $v_{\text {ine } 1}$.

From figure $1 b$, it appears that the inelastic peak decreases dramatically with increasing $v_{\text {inel }}$ for the $\mathrm{d}^{2} V / \mathrm{d} I^{2}$ curve, whereas it remains constant for $1 / \sigma \cdot \mathrm{d} \sigma / \mathrm{d} V$. The importance of this decrease may be calculated in the precise case of the electronic excitation of reference [3]. In that case, the reduced voltage $v_{\text {inel }}$ should be estimated. At the absolute voltage where the inelastic process appears $(1.15 \mathrm{~V})$ the conductance has become 17 times greater than at zero voltage, and to account for such an increase of $\sigma$, the parameter $V_{0}$ should be $0.28 \mathrm{~V}$. Then the reduced bias is $v_{\text {inel }}=4.1$.

At such a voltage, the ratio between the inelastic peaks on the two curves is 330 ! This clearly shows that the function $1 / \sigma . \mathrm{d} \sigma / \mathrm{d} V$ is better adapted to detecting such phenomena than $\mathrm{d}^{2} V / \mathrm{d} I^{2}$. It should be noticed that the

$$
\frac{\mathrm{d}^{2} I}{\mathrm{~d} V^{2}}=\frac{\mathrm{d} \sigma}{\mathrm{d} V}
$$

function would also give large inelastic peaks but on a baseline that would increase unpleasantly.

\subsection{Principle of A SyStem to obtain $\frac{1}{\sigma} \frac{\mathrm{d} \sigma}{\mathrm{d} V} \cdot$ - One}

means of studying the derivative $1 / \sigma . \mathrm{d} \sigma / \mathrm{d} V$ is described in reference [5] : an ordinary constant current modulation method produces the two characteristics $\mathrm{d} V / \mathrm{d} I=1 / \sigma$ and $\mathrm{d}^{2} V / \mathrm{d} I^{2}$, and a computer deduces the quantity of interest from the relation :

$$
\frac{1}{\sigma} \frac{\mathrm{d} \sigma}{\mathrm{d} V}=-\left(\frac{\mathrm{d} I}{\mathrm{~d} V}\right)^{2} \cdot \frac{\mathrm{d}^{2} V}{\mathrm{~d} I^{2}} .
$$

This method is not adapted to the problem of junctions whose conductance has increased by a large amount, because it multiplies a very small function $\mathrm{d}^{2} V / \mathrm{d} I^{2}$ (see Fig. 1) by a very large one $(\mathrm{d} I / \mathrm{d} V)^{2}$. Noise will largely affect the determination of the small quantity and the signal-to-noise ratio of the product will be poor.

A system which would give $\frac{1}{\sigma} \frac{\mathrm{d} \sigma}{\mathrm{d} V}$ at the output of the detector would be more suitable. Figure 2 shows a rather general diagram of a system built to give a

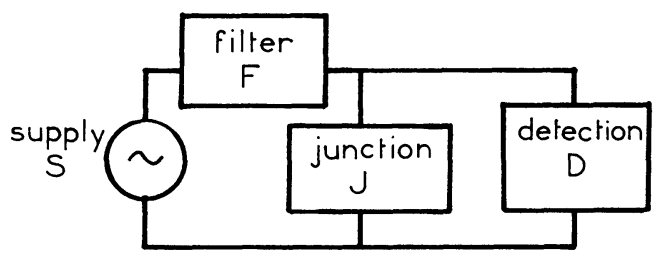

FIG. 2. - Diagram of a derivative system.

characteristic of a junction. It is made with different elements : a supply (S) that provides the bias and a modulation voltage at frequency $\omega$, a filter $(F)$, the junction ( $\mathrm{J}$ ) and the detection (D) which measures the harmonic of interest $(\omega, 2 \omega, 3 \omega, \ldots)$. If the voltage drop accross the element $\mathrm{k}$ is noted $V^{\mathrm{k}}(t)$ and the corresponding current $\mathrm{I}^{\mathrm{k}}(t)$, there are relations between their Fourier coefficients of the type : 
$V_{\omega}^{\mathrm{F}}=Z_{\omega}^{\mathrm{F}} I_{\omega}^{\mathrm{F}}, \quad$ where $Z_{\omega}^{\mathrm{F}}$ is the impedance of

$$
\begin{aligned}
I^{\mathrm{J}}(t) & =I_{0}^{\mathrm{J}}+I_{\omega}^{\mathrm{J}} \cos \omega t+I_{2 \omega}^{\mathrm{J}} \cos 2 \omega t \\
& =I\left(V_{0}^{\mathrm{J}}+V_{\omega}^{\mathrm{J}} \cos \omega t+V_{2 \omega}^{\mathrm{J}} \cos 2 \omega t\right),
\end{aligned}
$$

for the junction $\left(^{1}\right)$.

The different unknown quantities $I_{0, \omega, 2 \omega}^{\mathrm{k}}$ and $V_{0, \omega, 2 \omega}^{\mathrm{k}}$ can be calculated from the supply voltages and the impedances of the elements by using :

$$
\begin{aligned}
& \left.V_{\omega}^{\mathrm{S}}+V_{\omega}^{\mathrm{F}}+V_{\omega}^{\mathrm{J}}=0\right\} \\
& \cdots
\end{aligned}
$$

It can be seen that the second harmonic of the voltage across the junction is proportional to the quantity of interest :

$$
V_{2 \omega}^{\mathrm{J}} \simeq-\left(\frac{V_{\omega}^{S}}{2}\right)^{2} \cdot \frac{1}{\sigma} \frac{\mathrm{d} \sigma}{\mathrm{d} V},
$$

under the following conditions :

$$
\left.\begin{array}{c}
R \ll Z_{2 \omega}^{\mathrm{D}} \\
Z_{\omega}^{\mathrm{F}} \ll R Z_{2 \omega}^{\mathrm{F}}
\end{array}\right\},
$$

where $R$ is the junction dynamical resistance.

Remark. - The conditions

$$
\left.\begin{array}{rl}
Z_{2 \omega}^{\mathrm{D}} & \ll R \ll Z_{\omega}^{\mathrm{D}} \\
Z_{\omega}^{\mathrm{F}} & \ll R \ll Z_{2 \omega}^{\mathrm{F}}
\end{array}\right\}
$$

would give for the voltage across the junction :

$$
V_{2 \omega}^{\mathrm{J}}=-\left(\frac{V_{\omega}^{\mathrm{S}}}{2}\right)^{2} Z_{2 \omega}^{\mathrm{D}} \cdot \frac{\mathrm{d} \sigma}{\mathrm{d} V}
$$

which is an interesting quantity but corresponds to a smaller signal which would be more difficult to detect.

$$
\text { 2.3 Circuitry fOR } \frac{1}{\sigma} \frac{\mathrm{d} \sigma}{\mathrm{d} V} \text {. - The a. c. and d. c. }
$$
parts of the circuitry are shown in figures $3 a$ and $3 b$, they deserve a few comments :

- In order to avoid problems due to frequency shifts between the supply oscillator and the tuned circuits, their quality factors $(Q)$ are never greater than 20. Relation (5), which can then be written as :

$$
\frac{L_{1} \omega}{Q} \ll R \ll 2 Q L_{1} \omega,
$$

is rather severe and gives a narrow domain for junction resistances. We have therefore made several a. c. circuits in plug-in units (with different values for

(1) It can be shown that, in the case studied, there is no phase shift.

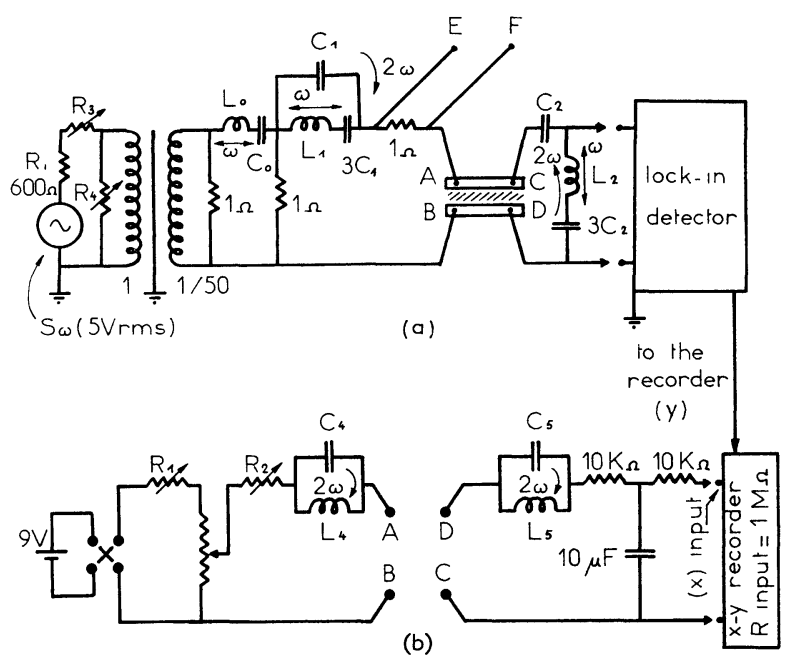

FIG. 3. - Circuitry to plot the $1 / \sigma . \mathrm{d} \sigma / \mathrm{d} V$ characteristic of a junction : a) a.c. elements, b) d.c. elements. For junctions whose resistances are in the range $50 \Omega-2 \mathrm{k} \Omega$ the values of the inductance and quality factors are :

$$
\begin{aligned}
& L_{0}=6 \mathrm{mH}, Q_{0}^{\omega}=10 ; L_{1}=35 \mathrm{mH}, Q_{1}^{\omega}=20 \\
& Q_{1}^{2 \omega}=20 ; L_{2}=8.5 \mathrm{H}, Q_{2}^{\omega}=10, Q_{2}^{2 \omega}=10 ; \\
& L_{4}=100 \mathrm{mH}, Q_{4}^{2 \omega}=10 ; L_{5}=1 \mathrm{H}, Q_{5}^{2 \omega}=10 .
\end{aligned}
$$

$\left.L_{1}\right)$ to allow the choice of that which is adapted to the junction resistance.

- At the detection, we have to increase the second harmonic-to-fundamental ratio in order to prevent the saturation of the lock-in. This is usually [6] done with a single tuned circuit, but here a doubly tuned circuit is used to decrease the first harmonic and enhance the second. This allows the use of an even lower $Q$ for a better ratio improvement.

The signal at the output of the detection filter can be expressed as :

$$
V_{2 \omega}^{\text {out }}=0.19 Q_{2 \omega}^{\mathrm{D}}\left(V_{\omega}^{\mathrm{S}}\right)^{2} \cdot \frac{1}{\sigma} \frac{\mathrm{d} \sigma}{\mathrm{d} V}
$$

where $Q_{2 \omega}^{\mathrm{D}}$ is the quality factor of the detection filter at frequency $2 \omega$.

- The second derivative measurements are not usually calibrated, and when quantitative values are required a first derivative $\sigma(v)$ is taken from the voltage between points $\mathrm{E}$ and $\mathrm{F}$, and used to calibrate the $1 / \sigma . \mathrm{d} \sigma / \mathrm{d} V$ curve.

- It sould be noted that in the d. c. circuitry, the $2 \omega$ tuned circuits provide the required high impedances at that frequency for the elements connected in parallel with the junction. At the d. c. detection, an additional low-pass filter prevents the modulation voltage across the junction from disturbing the $\mathrm{X}-\mathrm{Y}$ recorder or any noise in the recorder from going to the junction. It makes a calibrated weakening of the measured bias $(-2 \%)$.

3. Third derivative $\frac{d}{d V}\left(\frac{1}{\sigma} \frac{d \sigma}{d V}\right) \cdot-3.1$ USEFULNESS OF A THIRD DERIVATIVE. - As has already been 
mentioned, when the conductance of a junction increases rapidly with the voltage, the second derivative has a large base-line and it becomes difficult to distinguish small peaks. A further derivation will show up any very weak, but rather abrupt, detail in the curve as was shown in reference [3].

3.2 Different TYPES OF THIRD DERIVATIVE. - As in the case of the second derivative, this question is of importance. For instance, the derivative used in reference [3] :

$$
\frac{\mathrm{d}}{\mathrm{d} t}\left(\frac{1}{\sigma} \frac{\mathrm{d} \sigma}{\mathrm{d} V}\right)
$$

is easily obtained with a differential operational amplifier, but it decreases rapidly at high bias and then loses much of its interest.

In order to obtain a third derivative of the $I-V$ curve, the simplest technique would be to detect the third harmonic of the modulation, but one has to be very careful of cross terms : in general, the third harmonic is not proportional to the third derivative. An alternative solution is proposed that uses a double modulation technique and gives the function

$$
\frac{\mathrm{d}}{\mathrm{d} V}\left(\frac{1}{\sigma} \frac{\mathrm{d} \sigma}{\mathrm{d} V}\right)
$$

3.3 System. - Let us consider any system that gives a second derivative $y(V)$ at the output of a lock-in detector. If the bias voltage is slowly-modulated, the amplitude of the resulting modulation at the output is proportional to the derivative of $y$ with respect to $V$.

Using the system described in part 2 we shall apply a bias :

$$
V_{0}=V_{00}+V_{\Omega} \cos \Omega t .
$$

A first synchronous detection at frequency $2 \omega$, with time constant $\tau_{1}$, will give :

$$
\left(V_{\omega}\right)^{2} \cdot \frac{1}{\sigma} \frac{\mathrm{d} \sigma}{\mathrm{d} V}\left(V_{00}+V_{\Omega} \cos \Omega t\right),
$$

if the condition :

$$
\Omega \ll \frac{1}{\tau_{1}} \ll 2 \omega
$$

is fulfilled. A second lock-in detection at frequency $\Omega$, with a large time constant $\left(1 / \tau_{2} \ll \Omega\right)$, will give the required function :

$$
\left(V_{\omega}\right)^{2} V_{\Omega} \cdot \frac{\mathrm{d}}{\mathrm{d} V}\left(\frac{1}{\sigma} \frac{\mathrm{d} \sigma}{\mathrm{d} V}\right)
$$

The circuitry is that of figure 3 with the addition of the modulation supply described in figure 4 . In order to obtain the quantity (11) at the output of the first detector, the properties of the tuned filters at frequency $2 \omega$

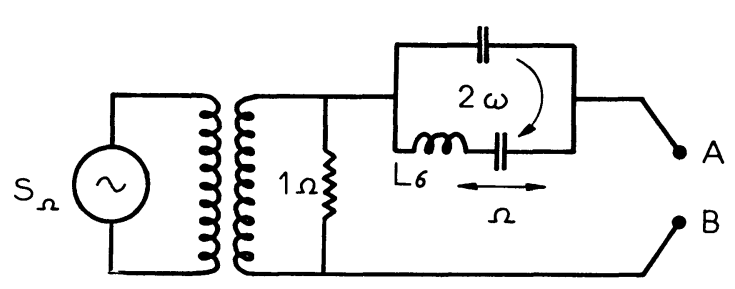

FIG. 4. - Low frequency modulation supply that should be added in parallel to the junction to get the third derivative. For $\omega=1 \mathrm{kHz}$ and $\Omega=20 \mathrm{~Hz}$, the value of the inductance $L_{6}$ is $0.15 \mathrm{H}$ and the quality factors are : $Q_{6}^{\omega}=2.5$ and $Q_{6}^{2 \omega}=20$. Then the supply impedance has the required properties for a wide range of junction resistances : $\frac{L \Omega}{Q \Omega}=6 \Omega \quad(\ll R)$ and

$$
2 Q_{2 \omega} . L \omega=40 \mathrm{k} \Omega(\gg R) \text {. }
$$

should remain the same in the band $[2 \omega \pm \Omega$ ]. With a quality factor $Q$, this requires :

$$
\Omega \ll \frac{2 \omega}{Q} .
$$

As the quality factors used vary from 10 to 20 we have chosen a modulation frequency $\Omega$ of $20 \mathrm{~Hz}$ for a main frequency $\omega$ at $1 \mathrm{kHz}$.

A $2 \omega$ and $\Omega$ tuned circuit makes this modulation supply a low impedance circuit at frequency $\Omega$ (to have relation (10)) and a high impedance one at frequency $2 \omega$ (relation (5)).

4. Tunneling experiments taking advantage of the third derivative. - The possibility of exciting electronic transitions by inelastic electron tunneling has been predicted since 1967 [2].

In 1972, we announced the observation of the effect in $\mathrm{Al}-\mathrm{Pb}$ junctions doped with organic molecules of

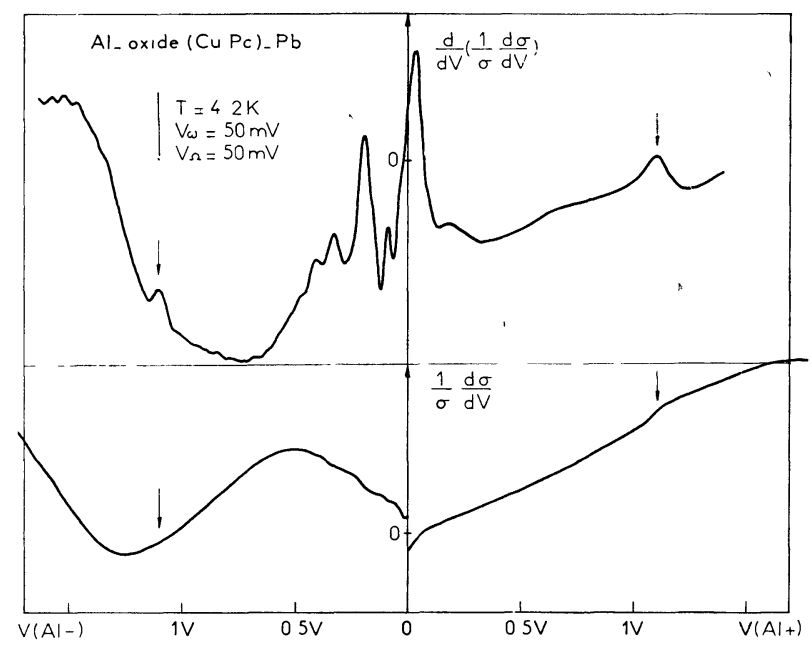

FIG. 5. - Second and third derivative of a Al-Pb junction doped with Copper Phthalocyanine ( $\mathrm{CuPc})$. On the second derivative $\frac{1}{\sigma} \frac{\mathrm{d} \sigma}{\mathrm{d} V}$ a structure can be seen at $1.15 \mathrm{~V}$ for the bias $\mathrm{Al}(+)$ but it escapes notice on the other polarity (one can see a minute flattening of the curve). On the third derivative, the corresponding structure is clearly observed for both polarities at exactly the same voltage, supporting the idea that it is due to an inelastic phenomenon. 
copper phthaloyanine $(\mathrm{CuPc})$. At that time, a peak could be observed in the plot of

$$
\frac{\mathrm{d}}{\mathrm{d} t}\left(\frac{1}{\sigma} \frac{\mathrm{d} \sigma}{\mathrm{d} V}\right)(V)
$$

at the voltage corresponding to the energy of an optically observed transition of $\mathrm{CuPc}$, but only in one polarity : $\mathrm{Al}(+)$. Some criticism had been opposed to the paper, remarking that inelastic peaks should be observed in both polarities. It was argued in the publication that in the other polarity, a very large base-line due to injection phenomena was hiding the peak. However, the observation of the effect for the bias $\mathrm{Al}(-)$ would have strongly confirmed the interpretation of the tunneling curves.

We have repeated the experiment using the third derivative

$$
\frac{\mathrm{d}}{\mathrm{d} V}\left(\frac{1}{\sigma} \frac{\mathrm{d} \sigma}{\mathrm{d} V}\right)(V)
$$

instead of that used in reference [3], in an attempt to extract the structure from the background. The resulting curves are reported in figure 5. A small peak $\left(^{2}\right)$ is indeed observed in the polarity $\mathrm{Al}(-)$, at the expected voltage $(1.1 \mathrm{~V})$. It should be noted that the second derivative characteristic has a minute flattening at this voltage but it completely escapes notice on that curve.

We think that this experiment supports the interpretation of reference [3] thus definitively establishing the observation of electronic transitions by tunneling spectroscopy, and that it also demonstrates the possibility of using a high order derivative to extract small structures from a large background in tunneling characteristics.

(2) One would expect the structure to be the derivative of $a$ peak, but probably, the asymmetry due to the strong variation of the base-line gives this aspect to the structures.

\section{References}

[1] Duke, C. B., Tunneling in Solids (ed. F. Seitz, Academic Press, N. Y.) 1969.

[2] Lambe, J. and Jaklevic, R. C., Phys. Rev. 165 (1968) 821.

[3] LéGer, A., Klein, J., Belin, M. and Défourneau, D., Solid State Commun. 11 (1972) 1331.
[4] Adler, J. G. and JACkson, J. E., Rev. Sci. Instrum. 37 (1966) 1049.

[5] Adler, J. G., Chen, T. T. and Straus, J., Rev. Sci. Instrum. 42 (1971) 362.

[6] Thomas, D. E. and Rowell, J. M., Rev. Sci. Instrum. 36 (1965) 1301. 